Piotr Janowiak', Alicja Siemińska', Maria Porzezińska', Żaneta Smoleńska², Hanna Suchanek², Ewa Jassem ${ }^{1}$

'Department of Pneumonology and Allergology, Medical University of Gdańsk, Poland

${ }^{2}$ Department and Clinic of Internal Medicine, Connective Tissue Diseases and Geriatrics, Medical University of Gdańsk, Poland

\title{
Difficulties in the treatment of recurring diffuse alveolar hemorrhage accompanying primary antiphospholipid syndrome: a case report and literature review
}

\begin{abstract}
Pulmonary embolism is the most common pulmonary manifestation of primary antiphospholipid syndrome (PAPS). However, PAPS may manifest in the respiratory system also due to non-thrombotic processes. In the following paper we present a case of PAPS-related diffuse alveolar hemorrhage (DAH). Because of sparse literature and a lack of randomized controlled trials, there are currently no recommendations regarding the optimal choice of steroid-sparing agent in treating PAPS-related DAH. In our patient, treatment with cyclophosphamide or mycophenolate mofetil along with low dose prednisone was ineffective, partially because of infectious complications, whereas addition of monthly intravenous immunoglobulin to mycophenolate mofetil and prednisone, appears to control the disease.
\end{abstract}

Key words: antiphospholipid syndrome; intravenous immunoglobulins; hemorrhage; mycophenolic acid; cyclophosphamide

Adv Respir Med. 2018; 86: 126-130

\section{Introduction}

Primary antiphospholipid syndrome (PAPS) is a systemic disease associated with recurring venous or arterial thrombosis and obstetric complications. The pathogenesis of these manifestations is related to the numerous effects of antiphospholipid antibodies (APLA) on hemostasis, with the most important effect attributed to anti-beta 2 glycoprotein 1 antibodies [1]. APLA are also reported to interfere with endothelial cell metabolism, platelet function, monocytes, and neutrophils. Furthermore, increases in oxidative stress and tissue factor generation along with activation of the complement system were noted [1]. PAPS, however, does not manifest exclusively with thrombotic symptoms. Other manifestations such as diffuse alveolar hemorrhage (DAH), Libman-Sacks endocarditis or chorea cannot be easily linked to the influence of APLA on blood coagulation [2]. Diffuse alveolar hemorrhage (DAH), which may be the first or isolated symptom in PAPS/APLA positive patients [3-6], is a clinical syndrome of multi-causal pathological background. As DAH symptoms are a result of an erythrocyte accumulation in the alveolar air space, most common features are diffuse bilateral airspace opacities on radiological examination, hypoxemic respiratory failure, anemia, and hemoptysis. Herein, we present a difficult to control case of PAPS-related DAH.

\section{Case report}

A 47-year-old Caucasian man was admitted to the hospital in December 2013 because of increasing dyspnea and hemoptysis, which had developed two days earlier. His medical history included PAPS, which had manifested as a deep venous thrombosis and pulmonary emboli, for

Address for correspondence: Piotr Janowiak, Department of Pneumonology and Allergology, Medical University of Gdańsk, Skłodowskiej-Curie 3A, 80-210 Gdańsk, Poland, tel. 00485834926 25, e-mail: 33033@gumed.edu.pl

DOI: 10.5603/ARM.2018.0020

Received: 01.05.2018

Copyright (C) 2018 PTChP

ISSN 2451-4934 
Table 1. Laboratory Tests in December 2013

\begin{tabular}{lc}
\hline CRP & $47.35 \mathrm{mg} / \mathrm{L}$ \\
PCT & $0.1 \mathrm{ng} / \mathrm{mL}$ \\
WBC & $7.85 \mathrm{G} / \mathrm{L}$ \\
HGB & $10.4 \mathrm{~g} / \mathrm{dL}$ \\
PLT & $250 \mathrm{G} / \mathrm{L}$ \\
INR & 2.23 \\
D-dimer & $217 \mu \mathrm{g} / \mathrm{L} \mathrm{FEU}$ \\
Cr & $1.65 \mathrm{mg} / \mathrm{dL}$ \\
BNP & $10 \mathrm{pg} / \mathrm{mL}$ \\
ANA-Hep2 & $1: 160$ \\
ANCA & $(-)$ \\
AECA & $(-)$ \\
Anti-GBM & $(-)$ \\
C3 & $0.85 \mathrm{~g} / \mathrm{L}$ \\
C4 & $0.06 \mathrm{~g} / \mathrm{L}$ \\
\hline
\end{tabular}

CRP: C-reactive protein; PCT: procalcitonin; WBC: white blood cells; HGB: hemoglobin; PLT: platelets; INR: international normalized ratio; Cr: creatinine; BNP: brain natriuretic peptide; ANA-Hep2: anti-nuclear antibodies Hep2; ANCA: anti-neutrophil cytoplasmic antibodies; AECA: anti-endothelial cell antibodies; Anti-GBM: anti-glomerular basement membrane antibodies; C3: complement component 3; C4: complement component 4

which warfarin was administered. Diagnosis met the revised Sapporo criteria [7]. Several months prior he had transient hearing loss, another possible PAPS manifestation [8], which resolved with glucocorticosteroids (GCS). Furthermore, he had a history of chronic renal disease and asthma. On admission, the patient was afebrile, normotensive (140/86 mm Hg), and not tachycardic (90 per minute). Auscultation of the lung revealed bilateral rales above the lower lungs, and wheezing. Arterial blood gas analysis showed hypoxemia $\left(\mathrm{pO}_{2}=57 \mathrm{~mm} \mathrm{Hg}\right)$. Laboratory results (Table 1) revealed increased C-reactive protein level $(47.35 \mathrm{mg} / \mathrm{L})$; however, the procalcitonin level and white blood cell count were within the normal range. Hemoglobin level was $10.4 \mathrm{~g} / \mathrm{dL}$. International normalized ratio (INR) was within the therapeutic range (2.23) and the D-dimer level was low $(217 \mu \mathrm{g} / \mathrm{L}$ FEU). The elevated creatinine levels $(1.46-1.74 \mathrm{mg} / \mathrm{dL})$ were stable in comparison to previous laboratory tests. Chest radiograph showed bilateral patchy alveolar infiltrates in the middle and lower parts of the lungs (Fig. 1). Echocardiogram did not reveal any structural abnormalities; right ventricular systolic pressure was not elevated, and the ejection fraction was $60 \%$. The high resolution computed tomography (HRCT) showed extensive bilateral ground-glass opacities (GGO) disseminated in the upper and

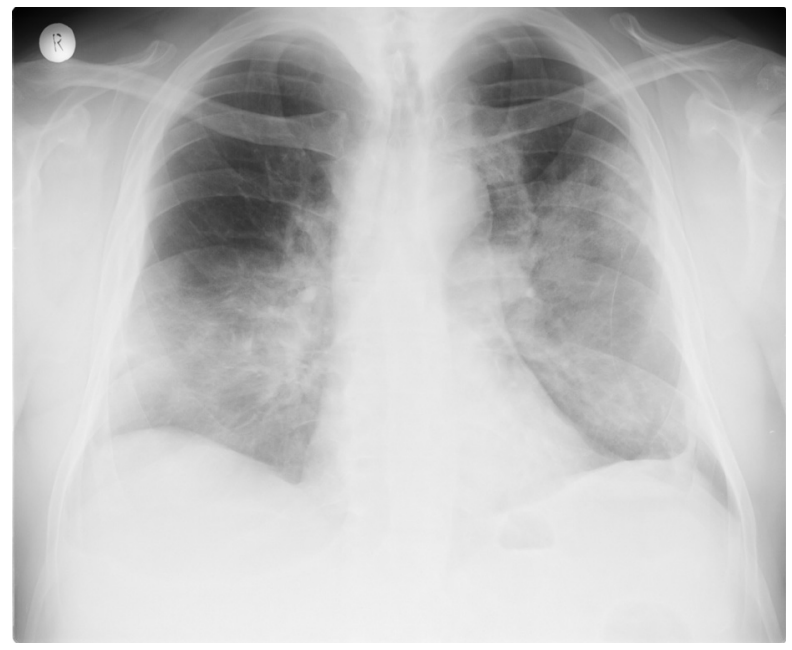

Figure 1. Chest radiogram obtained two days before admission in December 2013 showing patchy alveolar infiltrates in the middle and lower parts of the lungs

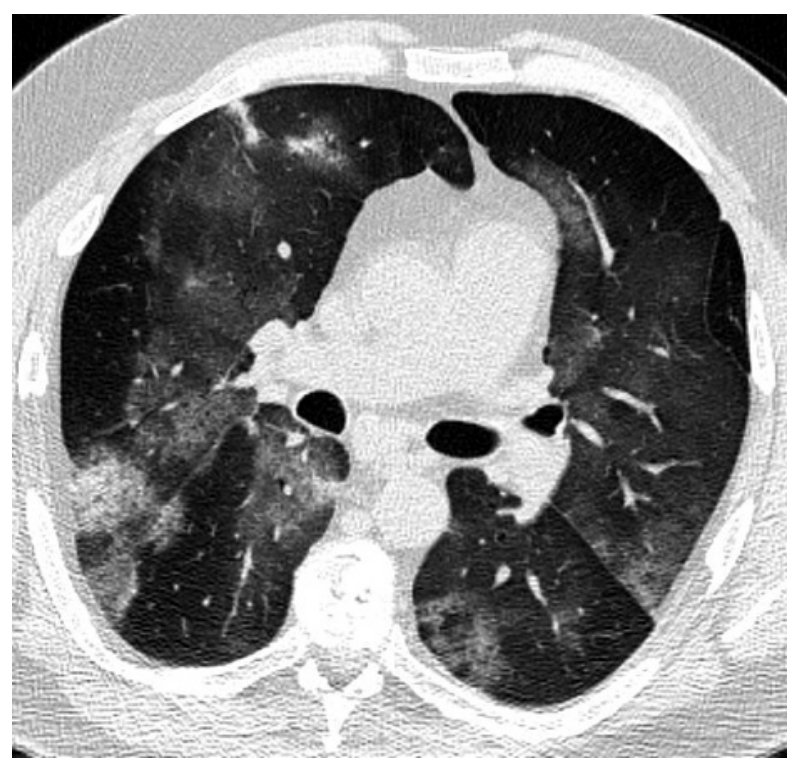

Figure 2. High resolution computed tomography at admission in December 2013 showing extensive bilateral ground-glass opacities (GGO)

lower lobes (Fig. 2). Bronchofiberoscopy did not reveal any pathology within the bronchial tree. Furthermore, the analysis of bronchoalveolar lavage fluid (BALF) samples for potential neoplasms or infections (including cytology, bacterial and fungal cultures), acid-fast bacilli smear and mycobacterial growth indicator tube cultures, immunofluorescence assay for Pneumocystis jiroveci cysts (PCPIF), and serum mannan and galactomannan antigen tests were negative. However, during BALF collection, progressively more hemorrhagic aliquots were obtained. BALF cytology revealed 
a high count of hemosiderin-laden macrophages (HLM), while the cell count differential was neutrophilic (49\%) supporting the diagnosis of DAH. Owing to the increased risk of complications, the patient was not referred to surgical lung biopsy for a pathological confirmation of the diagnosis. The treatment consisted of three methylprednisolone pulses with increasing dosage $(750 \mathrm{mg}, 1000 \mathrm{mg}$, $1000 \mathrm{mg}$ ), antibiotics (amoxicillin with clavulanic acid and clarithromycin), and antifungal agent (fluconazole). Simultaneously, anticoagulation therapy was temporarily discontinued. Methylprednisolone (MTP) was then switched to $80 \mathrm{mg}$ oral prednisone daily. The treatment resulted in significant improvement of dyspnea. To prevent thrombotic complications, warfarin was promptly reintroduced.

During hospitalization an extensive workup was performed to exclude non-PAPS-related potential causes of DAH, including systemic vasculitides and connective tissue diseases (CTD). Complement components $\mathrm{C} 3$ and $\mathrm{C} 4$ levels were slightly below the normal range $(0.85$ G/L; 0.06 G/L, respectively), whereas laboratory assays for antineutrophil cytoplasm antibodies (ANCA), antinuclear antibodies with HEp-2 cells (ANA-HEp2), anti-endothelial cell antibodies, and anti-glomerular basement membrane antibodies were negative. In addition, the patient did not report any clinical symptoms typical for CTD or systemic vasculitis. APLA titers were high (anticardiolipin and anti-beta2-glicoprotein antibodies values exceeded the upper reference limits). Retrospective analysis of the disease course revealed that he had previously experienced incidents of hemoptysis associated with multifocal GGO on HRCT with normal results on CT angiography, which had been interpreted at the time as due to an overdose of oral anticoagulants.

To maintain remission, cyclophosphamide (CYC) treatment along with prednisone in a tapering dosage was implemented. Within the first seven months of treatment, the patient received six $1.5 \mathrm{~g}$ CYC pulses every four weeks. In the eighth month of treatment, another acute DAH episode occurred, which was controlled by MTP (500 mg daily for 3 days) and intravenous immunoglobulin (IVIG; $50 \mathrm{~g}$ daily for 5 days). CYC treatment was replaced with MMF, along with prednisone, which provided only partial disease control during the following 29 months. DAH relapsed 3 times in this period, however, due to progressively milder course of DAH episodes (Table 2), with fourth one triggered by pneumonia, possibly a complication of high GCS dose (75 mg),

\section{Table 2. Course of treatment}

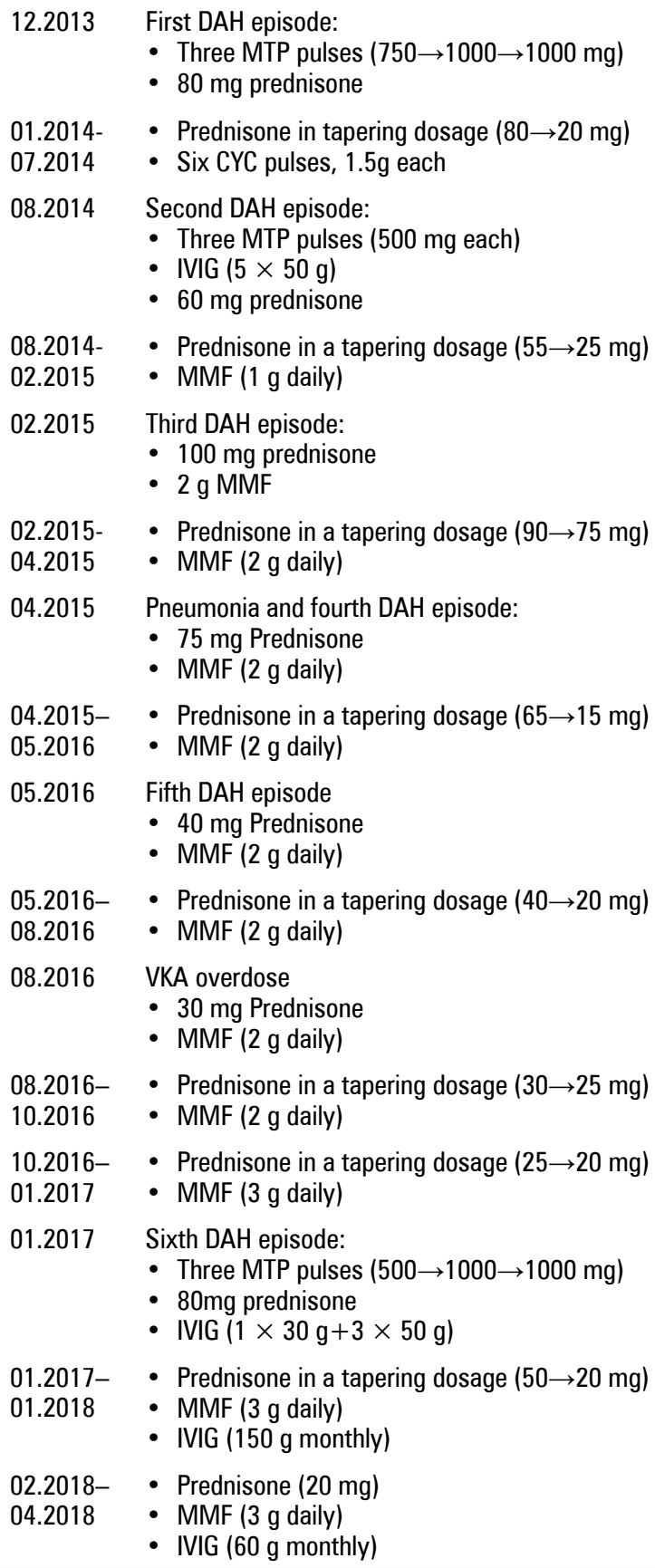

DAH: diffuse alveolar hemorrhage; MTP: methylprednisolone; CYC: cyclophosphamide; IVIG: intravenous immunoglobulin; MMF: mycophenolate mofetil

and with fifth one diagnosed accidentally after routine HRCT, MMF was sustained and its dose gradually increased up to 3 g daily. MMF treatment permitted significant GCS dose reduction (periodically down to $15 \mathrm{mg}$ ) but despite that patient experienced multiple, pathological rib and vertebrae fractures. Although bone density values were normal, glucocorticoid-induced osteoporosis and vitamin D insufficiency were recognized and proper treatment was commenced. 
In January 2017, while on 20 mg prednisone and 3 g MMF daily, patient experienced sixth DAH. Because of its severe course, including respiratory failure and hemoptysis, three pulses of MTP $(500,1000,1000 \mathrm{mg})$ were required along with IVIG (180 g). Afterwards monthly IVIG (150 g for 12 months, $60 \mathrm{~g}$ afterwards) was added to $3 \mathrm{~g}$ MMF along with prednisone in a tapering dosage which resulted in 16 months of disease stabilization.

\section{Discussion}

The etiology of DAH in PAPS is uncertain. One postulated mechanism involves capillaritis, which develops in a "two-hit" model [3], similar to a proposed mechanism of thrombosis in APS [9]. This hypothesis assumes that APLA, which influence the expression of pro-adhesive molecules on the surface of endothelial cells [1], are responsible for the "first hit". Other factors affecting endothelial cell activity, particularly those upregulating the adhesion molecules (such as infection or trauma), comprise the "second hit" resulting in neutrophil infiltration, vascular inflammation, and destruction of the alveolar septae. Unfortunately, not all patients who present with DAH in a PAPS/APLA setting actually exhibit neutrophilic vasculitis on pathologic examination. Yachoui et al. [4] published a case series report in which only one out of six patients subjected to lung biopsy showed neutrophilic infiltration, while the remaining five presented as bland hemorrhage [4]. Others have underlined [2] that neutrophilic interstitial infiltration alone, [3, $4,10,11]$ without perivascular or vascular infiltrates does not allow for recognizing vasculitis. However, lack of these features does not allow for a virtual ruling out of vasculitis because histological findings may vary depending on both the lung region and the activity of the disease [12]. On the other hand, to date, there have been only single case studies describing PAPS-related vasculitis in non-pulmonary systems [13]. Nevertheless, APLA-mediated pulmonary vasculitis may be a result of unique features of the lung, including a large endothelium surface area in relatively narrow capillaries that are positioned on a border with an environment rich in various stimuli [3].

Due to the inconsistencies in the vasculitis model, it has been suggested that DAH may be a result of complement system activation mediated by APLA [2], as it is postulated in obstetric APS [1]. This hypothesis is mainly based on studies on mice showing that complement fac- tor C2 mediates the neutrophilic infiltration and destruction of lung tissue after reperfusion of an ischemically injured gastrointestinal tract, most likely through the lectin pathway, of which C2 is an important part [2]. The lectin pathway might be activated by L-ficolin, which binds gram positive bacteria and is synthesized in the lung by type 2 pneumocytes and bronchial epithelium. Another study has shown that both murine and human APLA injected into complement receptor 2-deficient mice reconstitute ischemia/ /reperfusion damage locally in the intestines and remotely in the lungs [2].

There are certain similarities between our case and those previously described in the literature. Our patient is a male [3-6, 10, 11], in the fourth decade of life $[3,4,6,10,11]$, with a neutrophilic (49\%) BALF $[4,10]$ and high APLA titer $[3-5,10,11]$. Due to lack of the histopathological examination and normal bone density values, we cannot dismiss that pathological fractures, which our patient experienced, were a result of osteonecrosis, reported in other patients with PAPS as well [2]. Scheiman et al. [11] compared a group of 13 patients with pulmonary hemorrhage (PH) and secondary or primary APS to patients with APS without PH and showed that the former were more likely to experience non-thrombotic complications of APS, had a higher titer of APLA and a higher rate of pregnancy morbidity. Since the clinical presentation of non-thrombotic manifestations of PAPS is variable, Asherson et al. [2] speculated that there may be multiple subtypes of APLA, each with a different specificity, resulting in different symptoms.

The initial standard treatment of DAH includes GCS, typically either intravenous methylprednisolone or oral prednisone, depending on the severity of the episode. It should be noted that DAH symptoms may be mild enough to be overlooked in a proportion of patients. As reported by Yachoui et al. [4], such patients may experience complete and sustained remission without any immunosuppression. Moreover, not all patients will develop a relapse and require long-term treatment [6]. In our case, owing to recurrent disease and a high risk of new DAH episodes, we decided to start with CYC treatment. Although there are currently no randomized trials to support any recommendations regarding the choice of a steroid-sparing drug, CYC has been used effectively in vasculitides such as granulomatosis with polyangiitis and is suggested, along with rituximab, to be one of the most effective steroid-sparing agents in this setting [3, 4, 10]. However, CYC 
treatment failed to maintain remission in our patient. Switching to MMF also did not provide full control of the disease. Following the sixth DAH episode monthly IVIG was agreed upon. IVIG theoretically provides some advantages over immunosuppressant drugs. Its approved usage in primary and acquired immunodeficiencies can translate into protecting PAPS patients from infections which may, as in our patient, trigger DAH. Furthermore IVIG is an important part of catastrophic APS treatment and has been shown to be useful in preventing recurrent thrombotic incidents and obstetric complications of APS [14]. Despite this strong rationale behind its use, previous experiences with IVIG are mixed. Deane et al. [3] and Gertner et al. [6] described 3 patients who achieved disease control with IVIG, whereas other authors $[10,15]$ reported 6 patients who did not. In our patient adding IVIG allowed for 16 months of stabilization.

Other treatments explored in this setting, with mixed results, are: azathioprine $[4,5,10]$, methotrexate [4], hydroxychloroquine [3, 4] and plasmapheresis $[4,5,10]$.

\section{Conclusions}

Hemoptysis and dyspnea in PAPS require careful assessment. Pulmonary consultation along with bronchofiberoscopy should always be considered. A DAH diagnosis should be followed by an initial treatment consisting of high doses of GCS. There are currently no recommendations regarding the choice of steroid-sparing agent for long-term treatment. In our patient combined treatment, CYC or MMF with GCS, did not result in long-lasting remission and DAH reoccurred. As yet, the disease remains controlled after adding monthly IVIG to MMF and GCS. In our opinion it is worth considering adding IVIG to treatment of patients with recurrent infectious complications of immunosuppressive therapy.

\section{Acknowledgements}

Written, informed consent was obtained from the patient for publication of this case report and any accompanying data.

\section{Conflict of interest}

The authors declare no conflict of interest.

\section{References:}

1. Giannakopoulos B, Krilis SA. The pathogenesis of the antiphospholipid syndrome. N Engl J Med. 2013; 368(11): 1033-1044, doi: 10.1056/NEJMra1112830, indexed in Pubmed: 23484830.

2. Asherson RA, Cervera R, Shepshelovich D, et al. Nonthrombotic manifestations of the antiphospholipid syndrome: away from thrombosis? J Rheumatol. 2006; 33(6): 1038-1044, indexed in Pubmed: 16755649.

3. Deane KD, West SG. Antiphospholipid antibodies as a cause of pulmonary capillaritis and diffuse alveolar hemorrhage: a case series and literature review. Semin Arthritis Rheum. 2005; 35(3): 154-165, doi: 10.1016/j.semarthrit.2005.05.006, indexed in Pubmed: 16325656.

4. Yachoui R, Sehgal R, Amlani B, et al. Antiphospholipid antibodies-associated diffuse alveolar hemorrhage. Semin Arthritis Rheum. 2015; 44(6): 652-657, doi: 10.1016/j.semarthrit.2014.10.013, indexed in Pubmed: 25481816.

5. Koolaee RM, Moran AM, Shahane A. Diffuse alveolar hemorrhage and Libman-Sacks endocarditis as a manifestation of possible primary antiphospholipid syndrome. J Clin Rheumatol. 2013; 19(2): 79-83, doi: 10.1097/RHU.0b013e318278c577, indexed in Pubmed: 23340096.

6. Gertner E. Diffuse alveolar hemorrhage in the antiphospholipid syndrome: spectrum of disease and treatment. J Rheumatol. 1999; 26(4): 805-807, indexed in Pubmed: 10229400.

7. Miyakis S, Lockshin MD, Atsumi T, et al. International consensus statement on an update of the classification criteria for definite antiphospholipid syndrome (APS). J Thromb Haemost. 2006; 4(2): 295-306, doi: 10.1111/j.1538-7836.2006.01753.x, indexed in Pubmed: 16420554.

8. Wiles NM, Hunt BJ, Callanan V, et al. Sudden sensorineural hearing loss and antiphospholipid syndrome. Haematologica. 2006; 91(12 Suppl): ECR46, indexed in Pubmed: 17194652.

9. Meroni PL, Borghi MO, Raschi E, et al. Pathogenesis of antiphospholipid syndrome: understanding the antibodies. Nat Rev Rheumatol. 2011; 7(6): 330-339, doi: 10.1038/nrrheum.2011.52, indexed in Pubmed: 21556027.

10. Cartin-Ceba R, Peikert T, Ashrani A, et al. Primary antiphospholipid syndrome-associated diffuse alveolar hemorrhage. Arthritis Care Res (Hoboken). 2014; 66(2): 301-310, doi: 10.1002/acr.22109, indexed in Pubmed: 23983016.

11. Scheiman Elazary A, Cohen MJ, Aamar S, et al. Pulmonary hemorrhage in antiphospholipid antibody syndrome. J Rheumatol. 2012; 39(8): 1628-1631, doi: 10.3899/jrheum.120205, indexed in Pubmed: 22753647.

12. Asherson RA, Cervera R, Wells AU. Diffuse alveolar hemorrhage: a nonthrombotic antiphospholipid lung syndrome? Semin Arthritis Rheum. 2005; 35(3): 138-142, doi: 10.1016/j.semarthrit.2005.08.006, indexed in Pubmed: 16325654.

13. Lally L, Sammaritano LR. Vasculitis in antiphospholipid syndrome. Rheum Dis Clin North Am. 2015; 41(1): 109-23, ix, doi: 10.1016/j.rdc.2014.09.009, indexed in Pubmed: 25399943.

14. Tenti S, Cheleschi S, Guidelli GM, et al. Intravenous immunoglobulins and antiphospholipid syndrome: How, when and why? A review of the literature. Autoimmun Rev. 2016; 15(3): 226-235, doi: 10.1016/j.autrev.2015.11.009, indexed in Pubmed: 26656906.

15. Scheiman Elazary A, Klahr PP, Hershko AY, et al. Rituximab induces resolution of recurrent diffuse alveolar hemorrhage in a patient with primary antiphospholipid antibody syndrome. Lupus. 2012; 21(4): 438-440, doi: 10.1177/0961203311422713, indexed in Pubmed: 21993385. 